\title{
Nitroglycerin mediated dilation evaluated by ultrasound is associated with STWEAK in hemodialysis patients.
}

\author{
Crina Claudia Rusu¹, Liviu Ghervan², Simona Racasan³, Ina Kacso', Diana Moldovan', \\ Alina Potra ${ }^{1}$, Cosmina Bondor ${ }^{4}$, Florin Anton ${ }^{5}$, Ioan Mihai Patiu ${ }^{3}$, Mirela Gherman Caprioara ${ }^{1}$
}

${ }^{1}$ Department of Nephrology and Dialysis, "Iuliu Hatieganu" University of Medicine and Pharmacy, ${ }^{2}$ Institute of Clinical Urology and Renal Transplantation, "Iuliu Hatieganu" University of Medicine and Pharmacy, ${ }^{3}$ Nefromed Dialysis Center, ${ }^{4}$ Department of Informatics and Biostatistics, "Iuliu Hatieganu" University of Medicine and Pharmacy, $1^{\text {st }}$ Internal Medicine Department, "Iuliu Hatieganu” University of Medicine and Pharmacy, Cluj-Napoca, Romania

\begin{abstract}
Aims: The main cause of death in hemodialysis (HD) patients is cardiovascular disease. Ultrasound assessment of the brachial artery dysfunction is easily achievable and can non-invasively detect atherosclerosis in various stages. In HD patients the cardiovascular risk profile is different and the determinants of brachial arterial function can be distinct comparing with general population. The aim of the study is to assess the determinants of arterial brachial function (flow-mediated and nitroglycerinmediated dilation) evaluated by ultrasound in HD patients and their relation with tumor necrosis factor-like weak inducer of apoptosis (sTWEAK) described as atherosclerotic marker in chronic kidney disease patients. Material and methods: We conducted a cross-sectional observational study on 54 hemodialysis patients. We recorded clinical and biological data and we measured sTWEAK serum levels by ELISA. We evaluated the arterial brachial function by measurement of flow-mediated and nitroglycerin-mediated dilation, using B mode ultrasound. Results: The determinants of flow-mediated dilation were: Kt/V $(\mathrm{r}=0.47, \mathrm{p}<0.001)$, LDL-cholesterol $(\mathrm{r}=0.29, \mathrm{p}=0.04)$, and total cholesterol $(\mathrm{r}=0.31, \mathrm{p}=0.02)$. Flow-mediated dilation correlated with nitroglycerin-mediated dilation $(\mathrm{r}=0.70, \mathrm{p}<0.001)$. In multivariate analysis $\mathrm{kt} / \mathrm{V}$ was the only significant predictor for flow-mediated dilation $(\mathrm{p}=0.04)$. Nitroglycerin-mediated dilation correlates with sTWEAK $(\mathrm{r}=-0.30, \mathrm{p}=0.03)$, systolic blood pressure $(r=-0.28, p=0.04)$ and pulse pressure $(r=-0.31, p=0.02)$. In multivariate analysis sTWEAK was the only significant predictor for nitroglycerin-mediated dilation $(\mathrm{p}=0.04)$. Conclusions: The main determinant of nitroglycerin-mediated dilation was sTWEAK. In addition, decreased nitroglycerin-mediated dilation was associated with higher systolic blood pressure and pulse pressure. The main determinant of FMD was Kt/V. Increased flow-mediated dilation was associated with better dialysis efficiency and high total cholesterol and LDL-cholesterol.
\end{abstract}

Keywords: hemodialysis, vascular ultrasound, biomarkers

\section{Introduction}

In hemodialysis (HD) patients the main cause of death is cardiovascular disease. For this reason the detection of atherosclerosis (ATS) in preclinical phases using various techniques and biomarkers is a neces-

Received 27.08.2015 Accepted 20.10.2015

Med Ultrason

2016, Vol. 18, No 1, 57-63

Corresponding author: Ghervan Liviu

Institute of Clinical Urology

and Renal Transplantation

4-6 Clinicilor Str., 400006, Cluj-Napoca, Romania

Phone: 0040-745283705

E-mail:liviughervan@yahoo.com sity. Among these techniques, the endothelial function and vascular smooth muscle function evaluated with ultrasound flow-mediated dilation (FMD) and nitroglycerin-mediated dilation (NMD) are currently used. In addition, the panel of cardiovascular risk factors is different in HD patients, with more severe inflammation and ATS. Considering this, the parameters of FMD and NMD may be different compared to the general population.

Endothelial dysfunction is one of the initial changes in the development of ATS and an important predictor of cardiovascular disease in chronic kidney disease patients [1]. FMD has become the most widely used technique to measure endothelial function [2]. FMD is a non-invasive 
method, easily accessible, and with good correlation with invasive epicardial vascular function evaluation [3] but still requires better standardization [4].

Vascular smooth muscle dysfunction assessed by brachial NMD, was used initially as a control test for FMD measurements in order to differentiate endotheliumdependent from endothelium-independent vasodilation because both endogenous NO and administered nitroglycerin act on vascular smooth muscle cells. However, several studies have proved that both FMD and NMD are modified in subjects with cardiovascular risk factors and coronary heart disease $[5,6]$. In addition NMD proved to be a good predictor of cardiovascular mortality and morbidity both in the general population and in dialysis patients $[7,8]$. Impaired NMD is thought to be related with structural vascular alterations and smooth muscle cells dysfunction as a result of ATS [9] and can reflect arterial compliance [8].

Measurement of brachial FMD and NMD with ultrasound is generally achieved by grey scale, but in some studies Doppler exam is used to assess peak flow velocity (basal and after reactive hyperemia) [1].

Even if in principle FMD and NMD measurements seem simple, the procedures are technically challenging and require training and standardization. Most crucial are the study preparation, image acquisition, site selection, sphygmomanometer probe position, cuff occlusion time and correct description of the FMD response [4].

Mechanisms responsible for vascular disorders in HD patients are unclear, controversial, and presumed to be multifactorial. Endothelial dysfunction involves dysregulation of multiple pathways [10], one of them possibly mediated by tumor necrosis factor (TNF)-like weak inducer of apoptosis (TWEAK). This, a member of TNF family, is synthesized as a type II transmembrane glycoprotein and circulates in plasma as a soluble form (sTWEAK) [11]. This molecule can induces smooth muscle cells proliferation in the arterial wall [12] and seems to be involved in all pathogenetic phases of ATS [13].

A few studies found that in chronic kidney disease (CKD), sTWEAK correlates with brachial FMD and NMD. In HD patients the effect of sTWEAK on FMD and NMD was studied only in patients with no diabetes mellitus and no previous cardiovascular disease [10], with the emphasis mostly on endothelial dysfunction and less on muscular vascular dysfunction. Further studies are needed in order to assess the influence of this molecule on vascular dysfunction in HD patients.

The aim of the study is to assess the determinants of FMD and NMD in hemodialysis patients and to evaluate the relation between FMD, NMD, and sTWEAK described as atherosclerotic marker in CKD patients.

\section{Materials and methods}

\section{Patients:}

We conducted an analytical cross-sectional observational study, on a cohort of HD patients. All measurements were performed during a midweek non-dialysis day. Of the 110 patients on conventional HD treatment in Nefromed Dialysis Center Cluj, 54 patients met the inclusion criteria and also agreed to participate in this study in 2014. Inclusion criteria were: prevalent HD patients, age $>18$ years, duration of maintenance hemodialysis at least 6 months (HD vintage) and no residual renal function. We excluded patients with acute inflammation processes, terminal neoplasia, and viral hepatitis. All patients were on thrice weekly HD (4-5 h) regimen. Patients' demographics data, etiology of end stage renal disease (ESRD), HD vintage, and comorbidity conditions (diabetes, hypertension, smoking status, antihypertensive treatment, statins, antiplatelet therapy, erythropoietin treatment, intravenous iron) upon enrolment were obtained from medical documents. We registered clinical data: age, weight, height, systolic blood pressure (SBP), diastolic blood pressure (DBP) (predialysis values), triceps skinfold thickness (TST) $(\mathrm{mm})$, waist circumference $(\mathrm{WC})(\mathrm{cm})$. We also registered previous cardiovascular disease (angina or infarcts, coronary revascularization, stroke or documented peripheral arterial disease). We calculated body mass index (BMI) as BMI $=\left(\right.$ weight $(\mathrm{kg}) /$ height $^{2}\left(\mathrm{~m}^{2}\right)$ and pulse pressure $(\mathrm{PP})$ with formula: $\mathrm{PP}=\mathrm{SBP}-\mathrm{DBP}(\mathrm{mmHg})$.

All patients signed an informed consent before entering the study. Their privacy was respected. The study protocol conformed to the ethical guidelines and was approved by the University Ethics Committee, and was in accordance with the ethical standards of the World Medical Association, Declaration of Helsinki, revised in 2000, Edinburgh.

\section{Laboratory parameters}

All biochemical analyses were performed after an overnight fast between 7.00-9.00 a.m. always during a midweek dialysis day. Current measurements at the initiation of the study include serum electrolytes, albumin, predialysis creatinine, lipid profile (total cholesterol, triglycerides (TG) and HDL-cholesterol), C-reactive protein (CRP), intact parathormone (iPTH), hemoglobin, white blood cell (WBC). Pre- and post-dialysis urea levels were used to calculate Kt/V. LDL-cholesterol was calculated with Friedewald formula: LDL-cholesterol=total cholesterol-(HDL-chol+TG/5). STWEAK was determined with commercially available enzyme-linked immunosorbent assay kits (Bender MedSystem, Vienna, Austria kit for sTWEAK). The minimum detectable level 
of sTWEAK was $10 \mathrm{pg} / \mathrm{ml}$. Intra- and interassay coefficients of variation were $7.9 \%$ and $9.1 \%$ respectively.

\section{Ultrasonography}

Vascular measurements were performed using highresolution ultrasound: GE Logiq 3 (General Electric Company, Fairfield, CT, USA), with a 5-10 MHz linear transducer. The brachial artery in the arm without arteriovenous fistula was examined. The ultrasound examiner was blinded to other patient's data. All vasoactive medications were withheld 24 hours before the procedure and examination was performed after 14 hours of overnight fasting. After 15 minutes of rest before the examination started, the brachial artery diameter was assessed $5 \mathrm{~cm}$ above the antecubital fossa. Longitudinal images were obtained and the depth and gain setting were optimized to identify the anterior and posterior intimal interfaces between the lumen and vessel wall. The optimal grey scale image was obtained when the transducer was perpendicular on the vessel. Three adjacent measurements were recorded. In longitudinal view, both walls showed up distinctly over a certain range on a straight and nonbranching arterial segment. The transition from the intima to the lumen showed a weak signal, while the adventitia had a high amplitude signal. The media (between the 2 layers) had relatively low reflectivity and appeared as a hypo-echoic band on the images recorded with ultrasound systems with sufficient resolution [14] (fig 1). A pneumatic tourniquet was inflated $50 \mathrm{mmHg}$ above the systolic pressure for 5 minutes before deflating it. After 5 minutes the cuff was deflated and flow measurements were made at 1 minute and 10 minutes post deflation. After further 15 minute measurements were repeated and again 3 and 4 minutes after administration of sublingual $0.5 \mathrm{mg}$ nitroglycerin $[2,4]$. FMD was calculated as the percent change in brachial artery diameter post deflation compared with baseline resting diameters. NMD was calculated as the percent change in the brachial artery diameter post nitroglycerin administration compared with baseline resting diameters $[2,4,15]$.

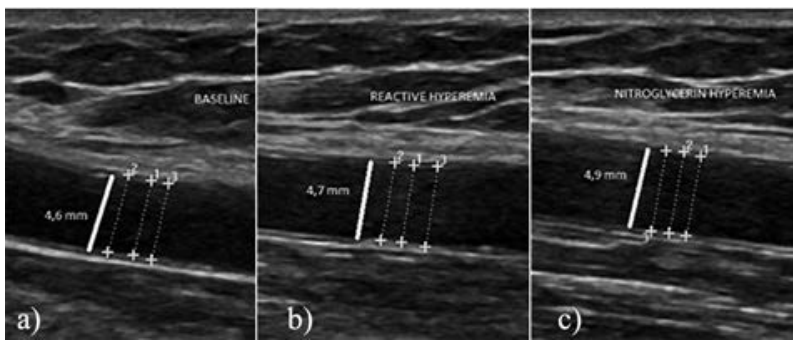

Fig 1. High resolution linear ultrasound measurement of brachial artery diameter a) before stimulus (baseline), b) $1 \mathrm{~min}$ after hyperemic stimulus (reactive hyperemia), and c) after nitroglycerin administration (nitroglycerin hyperemia).

\section{Dialysis prescription}

All patients were managed by nephrologists and were dialyzed with bicarbonate based dialysate, volumetric ultrafiltration control, single use synthetic (polysulphone) dialyzers and heparin as standard anticoagulant. Dialysis prescription was guided by a goal of achieving a value of $\mathrm{Kt} / \mathrm{V} \geq 1.4$

\section{Statistical analysis}

Data are presented as a mean \pm standard deviation (SD) for normally distributed variables or median (25th75th percentile) for non-normally distributed variables, or absolute or relative frequencies for nominal variables. The statistical comparison was performed using t-test for variables with normal distribution or the Mann-Whitney Rank Sum Test for the others. For identifying correlations between two continuous variables, Pearson's correlation coefficient or the Spearman correlation coefficient were used. The associations between NMD and FMD and oth-

Table I. Demographic, clinical and biochemical characteristics of patients $(n=54)$

\begin{tabular}{ll}
\hline Parameter & Value \\
\hline Age (years) & $60.4 \pm 1.7$ \\
HD vintage (months) & $59.4 \pm 5.8$ \\
Male n (\%) & $31(57.4)$ \\
Diabetes mellitus n (\%) & $9(16.7)$ \\
Hypertension n (\%) & $39(72.2)$ \\
SBP (mmHg) & $144.3 \pm 2.6$ \\
DBP (mmHg) & $72.9 \pm 1.6$ \\
PP (mmHg) & $71.4 \pm 2.6$ \\
Cardiovascular disease n (\%) & $12(22.2)$ \\
Smoking n (\%) & $8(14.8)$ \\
FMD (\%) & $8.9 \pm 1.2$ \\
NMD (\%) & $10.3 \pm 1.4$ \\
Body mass index (kg/m²) & $27.4(23.3-32.4)$ \\
Waist circumference (cm) & $99.1 \pm 2.3$ \\
Triceps skinfold thickness (mm) & $4(3-4)$ \\
Kt/V & $1.5 \pm 0.5$ \\
Total chol (mg/dl) & $172.6 \pm 5.9$ \\
LDL-chol (mg/dl) & $101.4 \pm 5.5$ \\
HDL-chol (mg/dl) & $38.5 \pm 1.3$ \\
Triglycerides (mg/dl) & $130.7(97.6-180.9)$ \\
Hemoglobin (g/l) & $11.4 \pm 0.1$ \\
Serum albumin (g/l) & $3.9 \pm 0.1$ \\
CRP (mg/dl) & $0.5(0.2-0.9)$ \\
Ca (mg/dl) & $8.9 \pm 0.1$ \\
P (mg/dl) & $4.7(4.1-6.1)$ \\
iPTH (pg/ml) & $270.5(146.9-658.1)$ \\
sTWEAK (pg/ml) & $3686.2(3086.1-4984.9)$ \\
\hline
\end{tabular}

Data are presented as mean \pm standard deviation or median (25th75th percentile); SBP: systolic blood pressure, DBP: diastolic blood pressure, PP: pulse pressure, FMD: flow-mediated dilation, NMD: nitroglycerin-mediated dilation, CRP: C-reactive protein, Ca: calcium, P: phosphate, iPTH: intact parathormone 
er clinical and biological parameters were analyzed with multivariate linear regression analysis (ENTER method - the model contains all the variables that in univariate analysis had $\mathrm{p} \leq 0.1)$. Standardized Beta and unstandardized $\mathrm{B}$ coefficient of regression equation, standard error and $95 \%$ confidence interval for $B$ were reported. $p \leq 0.05$ was considered statistically significant. Statistical analyses were performed using Statistica 7.0.

\section{Results}

\section{Patient characteristics}

The demographical, clinical and biological characteristics of the patients are presented in Table I. Forty five patients $(83.3 \%)$ had an arterio-venous fistula and the rest a semi-permanent transcutaneous access. The etiology of ESRD was chronic glomerulonephritis (21.5\%), diabetes $(16.7 \%)$, vascular nephropathy $(11.9 \%)$, tubulo-interstitial diseases $(11.4 \%)$, polycystic kidney disease $(7.2 \%)$, and unknown (31.4\%). We noted the treatment: $22 \%$ patients with statin, $17 \%$ patients under calcium-channel blockers, 37\% patients with renin angiotensin system inhibitors, $57 \%$ patients with beta blockers, $85 \%$ patients with erythropoietin, $46 \%$ patients with intravenous iron, and 35\% patients with antiplatelet therapy.

\section{Determinants of FMD in HD patients}

FMD significantly correlated with $\mathrm{Kt} / \mathrm{V} \quad(\mathrm{r}=0.47$, $\mathrm{p}<0.001)$, LDL-cholesterol $(\mathrm{r}=0.29, \mathrm{p}=0.04)$, total cholesterol $(\mathrm{r}=0.31, \mathrm{p}=0.02)$, with NMD $(\mathrm{r}=0.70, \mathrm{p}<0.001)$ and approached statistical significance in its correlation with SBP $(r=-0.25, p=0.07)$. Multivariate linear regression analysis (ENTER method) for FMD showed that Kt/V was the only predictor for FMD ( $\mathrm{p}=0.003)$ (Table II).

\section{Determinants of NMD in HD patients}

NMD significantly correlated with sTWEAK ( $\mathrm{r}=$ $-0.30, \mathrm{p}=0.03)$, SBP $(\mathrm{r}=-0.28, \mathrm{p}=0.04)$ and PP $(\mathrm{r}=-0.31$ $\mathrm{p}=0.02$ ). Multivariate linear regression analysis (ENTER method) for NMD showed that sTWEAK was the only predictor for NMD $(p=0.04)$ (Table II). We found no correlation of FMD with sTWEAK and no statistically significant variations of FMD or NMD depending on presence of diabetes mellitus, smoking, antihypertensive medication or statins.

\section{Discussions}

Vascular disorders are common in HD patients and have a major impact on survival. Vascular ultrasound evaluation may reveal endothelial dysfunction, vascular calcification, intima-media thickening, with important prognostic value in these patients [15]. Previous studies in HD patients assessed especially markers of endothelial dysfunction, considered as an early stage of ATS and were less focused on vascular smooth muscle dysfunction as marker of the severity of atherosclerosis. Accordingly, the main aim of our study was to assess the relationship between impaired NMD (a parameter that evaluates vascular smooth muscle dysfunction) and high levels of sTWEAK (a molecule shown to be involved in all ATS stages), a relationship that was confirmed by multivariate analysis. This association suggests that STWEAK might be a marker of vascular smooth muscle dysfunction in HD patients. It is known that sTWEAK induces smooth muscle cells proliferation in the arterial wall [12]. Consecutively a relative decrease in $\mathrm{NO}$ derived from nitroglycerin can occur [9] leading to impaired vascular smooth muscle function.

Whether low or high levels of sTWEAK are related to the NMD decrease in HD patients is not clear. In our study high levels of sTWEAK were associated with diminished NMD while others authors found that low sTWEAK levels were related to smaller NMD in HD patients [10]. In experimental studies increased sTWEAK in mice through genetic approaches resulted in the development of dilated cardiomiopathy with cardiac dysfunction and death [16], which indicates that high levels of sTWEAK are associated with cardiovascular pathology.

Table II. Multivariate linear regression analysis (ENTER method) for nitroglycerin-mediated dilation and flow-mediated dilation.

\begin{tabular}{|c|c|c|c|c|c|c|c|}
\hline & & \multicolumn{2}{|c|}{ Unstandardized Coefficients } & \multicolumn{2}{|c|}{$\begin{array}{l}\text { Standardized } \\
\text { Coefficients }\end{array}$} & \multicolumn{2}{|c|}{$\begin{array}{l}95 \% \text { Confidence Interval } \\
\text { for B }\end{array}$} \\
\hline & & B & Std. Error & Beta & $\mathbf{p}$ & Lower Bound & Upper Bound \\
\hline \multirow{4}{*}{ NMD } & SBP & -0.042 & 0.12 & -0.08 & 0.72 & -0.286 & 0.201 \\
\hline & PP & -0.03 & 0.119 & -0.075 & 0.74 & -0.28 & 0.201 \\
\hline & sTWEAK & -0.001 & 0.0006 & -0.281 & 0.04 & -0.002 & 0 \\
\hline & SBP & -0.001 & 0.066 & -0.002 & 0.988 & -0.133 & 0.132 \\
\hline \multirow{3}{*}{ FMD } & Total chol & -0.032 & 0.062 & -0.157 & 0.607 & -0.158 & 0.093 \\
\hline & LDL-chol & -0.075 & 0.064 & 0.337 & 0.245 & -0.053 & 0.203 \\
\hline & $\mathrm{Kt} / \mathrm{V}$ & 12.094 & 3.779 & 0.485 & 0.003 & 4.478 & 19.71 \\
\hline
\end{tabular}

FMD: flow-mediated dilation, NMD: nitroglycerin-mediated dilation, SBP: systolic blood pressure, PP: pulse pressure 
In addition, the differences between our results (high sTWEAK related with impaired NMD) and the results of other studies in HD patients (low sTWEAK related with impaired NMD) can have several explanations; first, by the characteristics of patients studied. Our group, comprising $17 \%$ patients with diabetes mellitus and $22 \%$ with previous CVD is different from those in previous studies that included only patients without diabetes mellitus and no previous CVD. It is known that in diabetes patients vascular smooth muscle function is significantly impaired by the diabetes itself [17]. Second, our patients might have genetic particularities which are responsible for circulating levels of sTWEAK. These particularities may cause defective shedding of sTWEAK in the bloodstream [10]. Third, in our study the association between high sTWEAK and impaired NMD might be due to the presence of a microenvironment which is either associated with low levels of sCD163 (sTWEAK scavenger) or with the overcoming of the capacity of sCD163 to bind and internalise sTWEAK. Moreover, all data available to date on sTWEAK come from observational studies and are somewhat contradictory. Patients with chronic diseases (including CKD) have been found to have lower values of sTWEAK than the general population [18-20], but in HD patients, higher levels of sTWEAK are associated with increased mortality [21].

Besides the relationship with sTWEAK, impaired NMD (increased vascular smooth muscle dysfunction) was associated in our study with high SBP and PP in agreement with previous studies in patients with normal renal function [9] and CKD patients. SBP and PP are markers of vascular stiffness, frequently present in HD patients, that can induce vascular smooth muscle dysfunction. In fact high blood pressure in HD patients was noted as an independent risk factor for vascular smooth muscle dysfunction [17].

Although we found a relation between NMD and STWEAK we did not find a correlation between FMD and sTWEAK in our group of patients. Others have noticed in HD and predialysis CKD patients a decrease in FMD accompanying the decrease in STWEAK $[10,22]$. A potential explanation for the difference may be related to the characteristics of the patients. As already mentioned in previous studies HD patients were included without diabetes mellitus and previous CVD or predialysis CKD patients which can have less severe vascular disorders. In addition, NMD was significantly correlated with the intima-media thickness of the brachial artery in other studies [9].

On the other hand, in our study the determinants of FMD were $\mathrm{Kt} / \mathrm{V}$ (as measure of dialysis efficiency), total cholesterol and LDL-cholesterol. Endothelial function improves as dialysis efficacy is improving, as has been previously shown [23], but increased HD vintage may predispose to ED [24]. Hemodialysis sessions diminish some of the risk factors associated with impaired endothelial function (excessive fluid and micro molecular toxins) and correct metabolic disorders in these patients, explaining the correlation between FMD and kt/V. However, in hemodialysis, the increased oxidative stress, inflammation and vascular calcifications may cause in time a greater arterial injury [24].

The correlation of LDL-cholesterol and total cholesterol with endothelial function was found by other authors, but in HD patients the type of correlation is different than that in the patients with normal renal function. Low levels of LDL-cholesterol and total cholesterol are associated with better endothelial function [25] in the general population. Surprisingly, in HD patients high levels of LDL-cholesterol are associated with better endothelial function (high FMD) [26], in agreement with our results. In fact in HD patients, as opposed to the patients with normal renal function, LDL and total cholesterol are mainly nutrition markers. It is known that dialysis patients with the lowest levels of LDL and total cholesterol are at very high risk for all-cause and cardiovascular mortality, likely because of the confounding by inflammation and malnutrition [27]. Moreover, it has been demonstrated that in CKD patients LDL-oxidation is not a major factor of endothelial dysfunction, more important are the inflammatory status [28], serum phosphate [29], or vitamin D levels [30].

The limits of our study are: first this is a cross-sectional observational study and we could evaluate only the associations between various parameters and not the pathogenetic mechanisms. The second limitation of our study is the relatively small cohort, while more extensive studies might assess the role of sTWEAK in evaluating the severity of vascular dysfunction and cardiovascular prognosis.

In conclusion the main determinant of NMD was sTWEAK. The correlation between STWEAK and NMD in our study suggests novel links between sTWEAK and vascular smooth muscle dysfunction in HD patients. Consequently, measuring STWEAK (as a predictor of vascular smooth muscle dysfunction) might contribute to better understanding the cardiovascular and survival prognosis in HD patients. Moreover, ultrasound assessment of brachial artery reactivity proves once again to be a readily available method that can improve the knowledge of the $\mathrm{CV}$ prognosis in HD patients. We consider that these methods can be used in clinical practice. Practitioners may use brachial artery FMD and NMD to as- 
sess response to therapy and to individualize patient risk modification programs.

Acknowledgements: This paper was published under the framework of the European Social Fund, Human Resources Development Operational Programme 20072013, project no. POSDRU/159/1.5/S/138776.

\section{Conflict of interest: none}

\section{References}

1. Lee MJ, Han SH, Lee JE, et al. Endothelial dysfunction is associated with major adverse cardiovascular events in peritoneal dialysis patients. Medicine (Baltimore) 2014 Sep; 93: e73.

2. Celermajer DS, Sorensen KE, Gooch VM, et al. Non-invasive detection of endothelial dysfunction in children and adults at risk of atherosclerosis. Lancet 1992; 340: 11111115.

3. Anderson TJ, Uehata A, Gerhard MD, et al. Close relation of endothelial function in the human coronary and peripheral circulation. J Am Coll Cardiol 1995; 26: 1235-1241.

4. Flammer AJ, Anderson T, Celermajer DS, et al. The assessment of endothelial function: from research into clinical practice. Circulation 2012; 126: 753-767.

5. Raitakari OT, Seale JP, Celermajer DS. Impaired vascular responses to nitroglycerin in subjects with coronary atherosclerosis. Am J Cardiol 2001; 87: 217-219.

6. Järvisalo MJ, Lehtimäki T, Raitakari OT. Determinants of arterial nitrate-mediated dilation in children: role of oxidized low-density lipoprotein, endothelial function, and carotid intima-media thickness. Circulation 2004; 109: 2885-2889.

7. Akamatsu D, Sato A, Goto H, et al. Nitroglycerin-mediated vasodilation of the brachial artery may predict long-term cardiovascular events irrespective of the presence of atherosclerotic disease, J Atheroscler Thromb 2010; 17: 12661274.

8. Dalton BS, Fassett RG, Geraghty DP, De Ryke R, Coombes JS. Vascular function and mortality in haemodialysis patients: a pilot study. Arch Cardiovasc Dis 2011; 104: 518523.

9. Maruhashi T, Soga J, Fujimura N, et al. Nitroglycerineinduced vasodilation for assessment of vascular function: a comparison with flow-mediated vasodilation. Arterioscler Thromb Vasc Biol 2013; 33: 1401-1408.

10. Yilmaz MI, Carrero JJ, Ortiz A, et al. Soluble TWEAK plasma levels as a novel biomarker of endothelial function in patients with chronic kidney disease. Clin J Am Soc Nephrol 2009; 4: 1716-1723.

11. Blanco-Colio LM, Martín-Ventura JL, Carrero JJ, et al. Vascular proteomics and the discovery process of clinical biomarkers: The case of TWEAK. Proteomics Clin Appl 2011; 5: 281-288.
12. Kim SH, Kang YJ, Kim WJ, et al. TWEAK can induce proinflammatory cytokines and matrix metalloproteinase-9 in macrophages. Circ J 2004; 68: 396-399.

13. Blanco-Colio LM. TWEAK/Fn14 Axis: A Promising Target for the Treatment of Cardiovascular Diseases. Front Immunol 2014; 5: 3 .

14. Stoner L, Sabatier MJ. Use of ultrasound for non-invasive assessment of flow-mediated dilation. J Atheroscler Thromb 2012; 19: 407-421.

15. Ghiadoni L, Faita F, Salvetti M, et al. Assessment of flowmediated dilation reproducibility: a nationwide multicenter study. J Hypertens 2012; 30: 1399-1405.

16. Ortiz A, Sanz AB, Munoz García B, et al. Considering TWEAK as a target for therapy in renal and vascular injury. Cytokine Growth Factor Rev 2009; 20: 251-258.

17. Rus R, Buturović-Ponikvar J. Intima media thickness and endothelial function in chronic hemodialysis patients. Ther Apher Dial 2009; 13: 294-299.

18. Díaz-López A, Bulló M, Chacón MR, et al. Reduced circulating sTWEAK levels are associated with metabolic syndrome in elderly individuals at high cardiovascular risk. Cardiovasc Diabetol 2014; 13: 51.

19. Vendrell J, Chacón MR. TWEAK: A New Player in Obesity and Diabetes. Front Immunol 2013; 4: 488.

20. Kralisch S, Ziegelmeier M, Bachmann A, et al. Serum levels of the atherosclerosis biomarker sTWEAK are decreased in type 2 diabetes and end-stage renal disease. Atherosclerosis 2008; 199: 440-444.

21. Carrero JJ, Ortiz A, Qureshi AR, et al. Additive effects of soluble TWEAK and inflammation on mortality in hemodialysis patients. Clin J Am Soc Nephrol 2009; 4: 110-118.

22. Yilmaz MI, Sonmez A, Ortiz A, et al. Soluble TWEAK and PTX3 in nondialysis CKD patients: impact on endothelial dysfunction and cardiovascular outcomes. Clin J Am Soc Nephrol 2011; 6: 785-792.

23. McGregor DO, Buttimore AL, Lynn KL, Yandle T, Nicholls MG. Effects of long and short hemodialysis on endothelial function: a short-term study. Kidney Int 2003; 63: 709-715.

24. Li X, Li L, Fang S, Xu Y. Effects of hemodialysis on brachial artery endothelial function: a clinical study.J Ultrasound Med 2012; 31: 1783-1787.

25. Delles C, Dymott JA, Neisius U, et al. Reduced LDL-cholesterol levels in patients with coronary artery disease are paralleled by improved endothelial function: An observational study in patients from 2003 and 2007. Atherosclerosis 2010; 211: 271-277.

26. Orasan R, Racasan S, Awon R, Patiu IM, Kacso I, Moldovan $\mathrm{D}$. Endothelial dysfunction is associated with visfatin and oxidative stress in hemodialysis patients, Studia Universitatis "Vasile Goldiş", Seria Ştiinţele Vieţii 2012; 22: 297-306.

27. Wanner C, Tonelli M; Kidney Disease: Improving Global Outcomes Lipid Guideline Development Work Group Members. KDIGO Clinical Practice Guideline for Lipid Management in CKD: summary of recommendation statements and clinical approach to the patient. Kidney Int 2014; 85: 1303-1309. 
28. Bolton CH, Downs LG, Victory JG, et al. Endothelial dysfunction in chronic renal failure: roles of lipoprotein oxidation and pro-inflammatory cytokines. Nephrol Dial Transplant 2001; 16: 1189-1197.

29. Watari E, Taketani Y, Kitamura T, et al. Fluctuating plasma phosphorus level by changes in dietary phosphorus intake induces endothelial dysfunction. J Clin Biochem Nutr 2015; 56: 35-42.

30. Chitalia N, Recio-Mayoral A, Kaski JC, Banerjee D. Vitamin D deficiency and endothelial dysfunction in non-dialysis chronic kidney disease patients. Atherosclerosis 2012; 220: 265-268. 